\title{
Pectoral Fin Anomalies in $t b x 5 a$ Knockdown Zebrafish Embryos Related to the Cascade Effect of N-Cadherin and Extracellular Matrix Formation
}

\author{
Jenn-Kan Lu ${ }^{1, \dagger}$, Tzu-Chun Tsai ${ }^{2,3}$, Hsinyu Lee ${ }^{4}{ }^{\oplus}$, Kai Hsia ${ }^{2,4,5}$, Chih-Hsun Lin ${ }^{2,6}$ \\ and Jen-Her $\mathrm{Lu}$ 2,5,*,+(i) \\ 1 Laboratory of Molecular Biology, Institute of Aquaculture, National Taiwan Ocean University, \\ Keelung 20224, Taiwan \\ 2 Institutes of Clinical Medicine; Department of Surgery and Pediatrics, School of Medicine, \\ National Yang-Ming University, Taipei 11221, Taiwan \\ 3 Department of Pediatrics, Taoyuan General Hospital, Ministry of Health and Welfare, Taoyuan 33004, Taiwan \\ 4 Department of Life Science, National Taiwan University, Taipei 10617, Taiwan \\ 5 Department of Pediatrics, Taipei Veterans General Hospital, No. 201, Section 2, Shih-Pei Rd., Beitou, \\ Taipei 11217, Taiwan \\ 6 Department of Plastic and Reconstructive surgery, of Surgery, Taipei Veterans General Hospital, No. 201, \\ Section 2, Shih-Pei Rd., Beitou, Taipei 11217, Taiwan \\ * Correspondence: jenherlu@gmail.com \\ + These authors contributed equally to this work.
}

Received: 27 May 2019; Accepted: 10 July 2019; Published: 12 July 2019

\begin{abstract}
Functional knockdown of zebrafish tbx5a causes hypoplasia or aplasia of pectoral fins. This study aimed to assess developmental pectoral fin anomalies in $t b x 5 a$ morpholino knockdown zebrafish embryos. The expression of cartilage-related genes in the $t b x 5 a$ morphant was analyzed by DNA microarray, immunostaining, and thin-section histology to examine the detailed distribution of the extracellular matrix (ECM) during different pectoral fin developmental stages. Chondrogenic condensation (CC) in the $t b x 5 a$ morpholino knockdown group was barely recognizable at $37 \mathrm{~h}$ postfertilization (hpf); the process from CC to endoskeleton formation was disrupted at $48 \mathrm{hpf}$, and the endoskeleton was only loosely formed at $72 \mathrm{hpf}$. Microarrays identified 18 downregulated genes in $t b x 5 a$-deficient embryos, including 2 fin morphogenesis-related $(c x 43, b b s 7)$, 4 fin development-related (hoxc8a, hhip, axin1, ms xb), and 12 cartilage development-related (mmp14a, sec23b, tfap2a, slc35b2, dlx5a, dlx1a, tfap2b, fmr1, runx3, cdh2, lect1, acvr2a, mmp14b) genes, at 24 and $30 \mathrm{hpf}$. The increase in apoptosis-related proteins (BAD and BCL2) in the tbx5a morphant influenced the cellular component of pectoral fins and resulted in chondrocyte reduction throughout the different CC phases. Furthermore, tbx $5 a$ knockdown interfered with ECM formation in pectoral fins, affecting glycosaminoglycans, fibronectin, hyaluronic acid (HA), and N-cadherin. Our results provide evidence that the pectoral fin phenotypic anomaly induced by $t b x 5 a$ knockdown is related to disruption of the mesoderm and ECM, consequently interfering with mesoderm migration, CC, and subsequent endoskeleton formation.
\end{abstract}

Keywords: chondrogenic condensation; tbx $5 a ; \mathrm{N}$-cadherin; chondrogenesis; fibronectin; hyaluronic acid; endoskeleton formation

\section{Introduction}

Chondrogenesis is one of the earliest events in the morphogenesis of the pectoral fin [1]. The genetic pathways of the pectoral fin in zebrafish and forelimbs in tetrapods are highly conserved [2]. 
Holt-Oram syndrome is an inherited disorder in humans characterized by upper limb deformity and congenital heart disease, and is related to mutation of the $t b x 5 a$ (T-box transcription factor 5) gene [3-6]. However, the molecular and cellular processes underlying upper limb agenesis or hypoplasia in Holt-Oram syndrome remain unclear.

The $t b x 5 a$ gene is expressed in the early stage of pectoral fin development in zebrafish embryos $[7,8]$. The bilateral protrusions of the mesoderm in the lateral plate are pectoral fin precursor cells that form the pectoral fin buds in zebrafish. The expression of the $t b x 5 a$ gene in the lateral plate mesoderm at 10-15 h postfertilization (hpf) constitutes the earliest specific marker of pectoral fin development in zebrafish [9-11]. The signaling center of pectoral fin buds in zebrafish embryos is located in the apical ectodermal ridge (AER). Formation of the pectoral fin includes blastema formation, and pectoral fin bud formation in zebrafish embryos is initiated at $26 \mathrm{hpf}$ [12]. Mesenchymal cells cluster to form oval-shaped pectoral fin buds at $32 \mathrm{hpf}$. The formation of the AER is initiated at $36 \mathrm{hpf}$ via chondrogenic condensation (CC) [13].

The mesenchymal cell migration during fin formation, which plays a crucial role in the localization and timing of pectoral fin bud induction, depends on the expression of the $t b x 5 a$ gene [14-16]. The $t b x 5$ gene is essential for initiating AER signaling in mesenchymal cells until the end of pectoral fin bud formation at $48 \mathrm{hpf}$. Furthermore, tbx5a expression is maintained at a low level up to $96 \mathrm{hpf}$ [17-19]. Defects in the $t b x 5 a$ gene result in variable scapulocoracoid, endoskeleton disc, hypoplasia, and distal cleithrum dislocation defects. These limb bud-derived abnormalities consequently cause agenesis, shortening, or deformity of the pectoral fin $[9,18,20]$. The functional knockdown of zebrafish $t b x 5 a$ by morpholino (MO) administration results in a failure of mesodermal cell migration, which leads to the failure of initiating fin bud formation and suggests that $t b x 5 a$ functions very early in the pectoral fin induction pathway [14].

This study aims to assess cellular and extracellular matrix (ECM) components in the pectoral fin of $t b x 5 a$ gene knockdown zebrafish embryos. The expression of cartilage-related genes in $t b x 5 a$ gene knockdown zebrafish embryos was studied by DNA microarrays and immunohistology to examine the distributions of proteoglycans (PGs) and neural cadherin (N-cadherin) at different pectoral fin developmental stages.

\section{Materials and Methods}

\subsection{Animal Ethics}

Approval for this experiment was granted by the Animal Ethics Review Board of National Taiwan Ocean University Aquaculture (IACUC 105031, College of Life Science). Since zebrafish embryos under 7 days (168 hpf) are excluded from the definition of "vertebrate animals" provided by the review board, our study, which used zebrafish embryos under $96 \mathrm{hpf}$, was spared the regulation and review process required by the Basic Institutional Review Board (IRB).

\subsection{Maintenance and Breeding of Zebrafish and Embryo Collection}

We used the Zebrafish $\mathrm{AB}$ strain carrying a specific transgene ( $\mathrm{Tg}$, cmlc2::EGFP; cmlc2:H2AFZmCherry) $)^{\mathrm{cy} 3}$ in this study. The details of the maintenance and breeding of zebrafish and embryo collection have been previously published [21-23].

\subsection{Microinjections and Morpholino Treatment}

Total RNA was prepared from defective or normal embryos in each group (Invitrogen Corporation, Carlsbad, CA, USA). The MO antisense oligonucleotide $t b x 5 a$-MO (5'-GAAAGGTGTCTTC ACTGTCCGCCAT- $3^{\prime}$ ) was designed against the $t b x 5 a$ translational start site, and a mismatch- $t b x 5 a-\mathrm{MO}$ (5'-GTCTCTTGACTCTCCGCGATCTCGG-3') was designed as a control (Gene Tools LLC, Philomath, OR, USA). Wild-type (WT) embryos, primarily at the one-cell stage with an intact chorion, were injected with $19.4 \mathrm{ng} / 4.3 \mathrm{~nL}$ stock MO diluted in Danieau's buffer ( $n=50$ per group in triplicate). 
Three control groups, including (1) Control MO-injected group injected with the $3^{\prime}$ end of $t b x 5 a-\mathrm{MO}$, (2) Blank microinjection group, and (3) Uninjected group (no microinjections), were included to identify the specific effects of blocking $t b x 5 a$ mRNA translation with $t b x 5 a-\mathrm{MO}$ ( $n=50$ per group) (Supplementary Materials).

\subsection{Validation of the Efficacy, Traumatic Effects, and Off-Target Effects of tbx5a MO}

To rule out the possibility that pectoral fin anomalies were induced by microinjection or a nonspecific off-target effect of $t b x 5 a \mathrm{MO}$, we used early microinjection of missense $t b x 5 a$ (Control MO-injected group) into the 1-4 cell stage of WT embryos as a control. We used Western blot to validate the efficacy of $t b x 5 a \mathrm{MO}$. The off-target effect of microinjected $t b x 5 a \mathrm{MO}$ at the 1-4 cell stage was further evaluated by longitudinal follow-up studies. We used several indexes, including the longitudinal survival rate and heart rate follow-up, heart defect rate, and rate of trunk deformity (Supplementary Data 1).

\subsection{Histology}

At $48 \mathrm{hpf}$, the embryos were fixed in $4 \%$ paraformaldehyde, dehydrated using an ethanol series, cleared in xylene, and embedded in paraffin wax. Longitudinal sections were cut, dewaxed in xylene, and stained with hematoxylin and eosin (HE).

\subsection{Alcian Blue Staining}

Formaldehyde-fixed and paraffin-embedded tissue sections were processed in $70 \%$ ethanol for $3 \mathrm{~min}$ at room temperature and then incubated in 3\% acetic acid for $3 \mathrm{~min}$. The tissues were immersed in Alcian blue (ScienCell Research Lab, Carlsbad, CA, USA) solution (1\% Alcian blue in 3\% acetic acid) for $30 \mathrm{~min}$. After tissue sections were washed in running tap water for $1 \mathrm{~min}$ and rinsed in $\mathrm{diH}_{2} \mathrm{O}$ for $2 \mathrm{~min}$, the sections were dehydrated in two changes of $95 \%$ ethanol for $2 \mathrm{~min}$ each and cleared in xylene substitute (ScienCell Research Lab, CA, USA).

\subsection{Immunohistochemistry Assay}

At $30 \mathrm{hpf}$, embryos were fixed with $4 \%$ paraformaldehyde in phosphate-buffered saline (PBS). The deparaffinized sections $(3 \mu \mathrm{m})$ were incubated with target-purified rabbit primary antibodies against HA (AnaSpec Inc., Fremont, CA, USA) and fibronectin (AnaSpec Inc., Fremont, CA, USA), washed with PBS, and incubated with rhodamine-conjugated goat anti-rabbit immunoglobulin G (IgG). Control samples were processed in parallel, omitting the primary antibody. Confocal images were obtained using a Zeiss LSM 880 confocal microscope with a 20× objective. Zen image acquisition software (Carl Zeiss, Jena, Germany) was used to analyze images. Samples of the $t b x 5$ morphant group at specific stages of embryo development were detected by the argon laser at wavelengths of $488 \mathrm{~nm}$ and $530 \mathrm{~nm}$ with a 20x objective lens and compared to those of the Uninjected and Control MO injection groups. Samples were scanned by controlling the laser wavelength between $510 \sim 540 \mathrm{~nm}$ and 580 650 $\mathrm{nm}$ to yield green and red fluorescence, respectively. The optimal aperture (pinhole) was adjusted by the receiver range, noise ratio control of the detector (amplification offset and amplification gain), and laser beam intensity. The best fluorescence intensity balance of the tissue image capture resolution was set to $1024 \times 1024$ to minimize the impact of background fluorescence. The images were reconstructed and observed using LCS Lite software (version 2.0) (Leica, Bannockburn, IL, USA).

\subsection{RNA Isolation}

Total RNA was isolated from 50 embryos using a guanidine isothiocyanate-based TRIzol solution. RNA samples were resuspended in diethyl pyrocarbonate (DEPC)-treated water and quantified spectrophotometrically at $260 \mathrm{~nm}$. RNA quality was checked by $1.2 \%$ agarose gel electrophoresis after staining with $1 \mu \mathrm{g} / \mathrm{mL}$ ethidium bromide. The RNA stock solution was stored at $-80{ }^{\circ} \mathrm{C}$. 


\subsection{Microarray Analysis}

Microarray analysis (WT vs. MO) was performed to determine the effect of $t b x 5$ deficiency. RNA from the WT and MO groups was isolated at 24, 30, and $48 \mathrm{hpf}$ and purified using a RNeasy ${ }^{\circledR}$ Mini Kit (QIAGEN, Hilden, Germany). RNA quality was confirmed using an Agilent 2100 Bioanalyzer (Agilent Technologies, Santa Cruz, CA, USA). Purified RNA was reverse-transcribed into cDNA using SuperScript TM III RT (Invitrogen, Carlsbad, CA, USA). Before purifying and coupling with a fluorescent dye by indirect cDNA labeling using a zebrafish-specific microarray kit covering 43,663 gene transcripts (Invitrogen, Carlsbad, CA, USA), cDNA was hydrolyzed and neutralized using $\mathrm{NaOH}$ and $\mathrm{HCl}$. Then, the cDNA was pretreated with the GEx hybridization buffer HI-PRM (Agilent Technologies, Santa Cruz, CA, USA) before being transferred to hybridization chamber gasket slides for the hybridization reaction. The slides were scanned using a GenePix 4000B Axon Instruments scanner (Molecular Devices, Silicon Valley, CA, USA), and the data were analyzed using Genespring GX 10.0.2 (Agilent Technologies, Santa Cruz, CA, USA). All gene transcripts $(43,663)$ were screened at these three time points. All data were compliant with the Minimum Information about a Microarray Experiment guidelines, and the raw data were deposited into the Gene Expression Omnibus (GSE33965, NCBI tracking system \#16217606) as previously described $[17,18]$. Microarray data were analyzed based on gene expression data, expression ratios and normalized intensities. Housekeeping genes (w.r.t GAPDH) were used as a reference for normalization, and all other conditions were normalized with respect to the reference to obtain expression ratios. Each gene expression value in a single array experiment was divided by the mean expression values of these housekeeping genes. The expression of a certain gene in the Uninjected group was used as a reference and set to a value of 1 . If the expression level of the same gene in the tbx5a morphant group was more than 1.5-fold higher than that in the Uninjected group, the gene was marked as "upregulated". Conversely, if the expression level of the same gene in the $t b x 5 a$ morphant group were more than 1.5 -fold lower than that in the Uninjected group, the gene was marked as "downregulated". These comparisons were analyzed at 24,30, and $48 \mathrm{hpf}$. Pathway software was used to analyze fin morphogenesis and chondrogenesis. The expression level of each candidate gene that exceeded or fell below the threshold was further verified by quantitative RT-PCR at different developmental stages.

\subsection{Quantitative Reverse-Transcriptase Polymerase Chain Reaction ( $q P C R$ )}

Total RNA from 50 defective or normal embryos was prepared by the amplification of $3 \mu \mathrm{L}$ of first-strand cDNA (Invitrogen). The amplification primer for the $c d h 2$ mRNA was obtained from published sequences as follows: $c d h 2$ (F: 5'-TGG CAA GAG GAC AAG GCG AGG ACG A-3'; R: 5' GTG GGC AAT CAC TGG GTT GGG GCA-3'). The PCR conditions included denaturation at $95^{\circ} \mathrm{C}$ for $3 \mathrm{~min}$ followed by 50 cycles of amplification $\left(95^{\circ} \mathrm{C}\right.$ for $20 \mathrm{~s}, 59^{\circ} \mathrm{C}$ for $15 \mathrm{~s}$, and $72{ }^{\circ} \mathrm{C}$ for $20 \mathrm{~s}$ ).

\subsection{Statistical Analysis}

All experiments were repeated at least three times, and the data are shown as the mean \pm SD. The paired sample $T$ test was applied for statistical analysis between experimental groups, and $p<0.05$ was considered significant. One-way ANOVA with Duncan's post hoc multiple range test was used to compare data among more than two groups, and $p<0.05$ was considered significant. Statistical analysis was performed using IBM SPSS Statistics 19 (version 19; SPSS, Chicago, IL, USA).

\section{Results}

\subsection{Abnormal Phenotypes of Pectoral Fins}

After microinjection of $t b x 5 a \mathrm{MO}$, embryos showed developmental defects in the pectoral fin at $96 \mathrm{hpf}$. The pectoral fin in the $t b x 5 a$ morphant group showed unilateral or bilateral hypoplasia with a shortened length (Figure 1C-E). These abnormal hypoplastic limb buds exhibited outward flexion and/or were flat in shape (Figure 1A). The development of the limb bud was disrupted in most 
members of the $t b x 5 a$ morphant group. In the $t b x 5 a$ morphant group, $12 \%$ of the embryos showed normal fin development (6/50) at $48 \mathrm{hpf}, 10 \%$ (5/50) showed normal fin development at $72 \mathrm{hpf}$, and $8 \%$ $(4 / 50)$ showed normal fin development at $96 \mathrm{hpf}(n=50$ each, triplet) (Figure 1F).
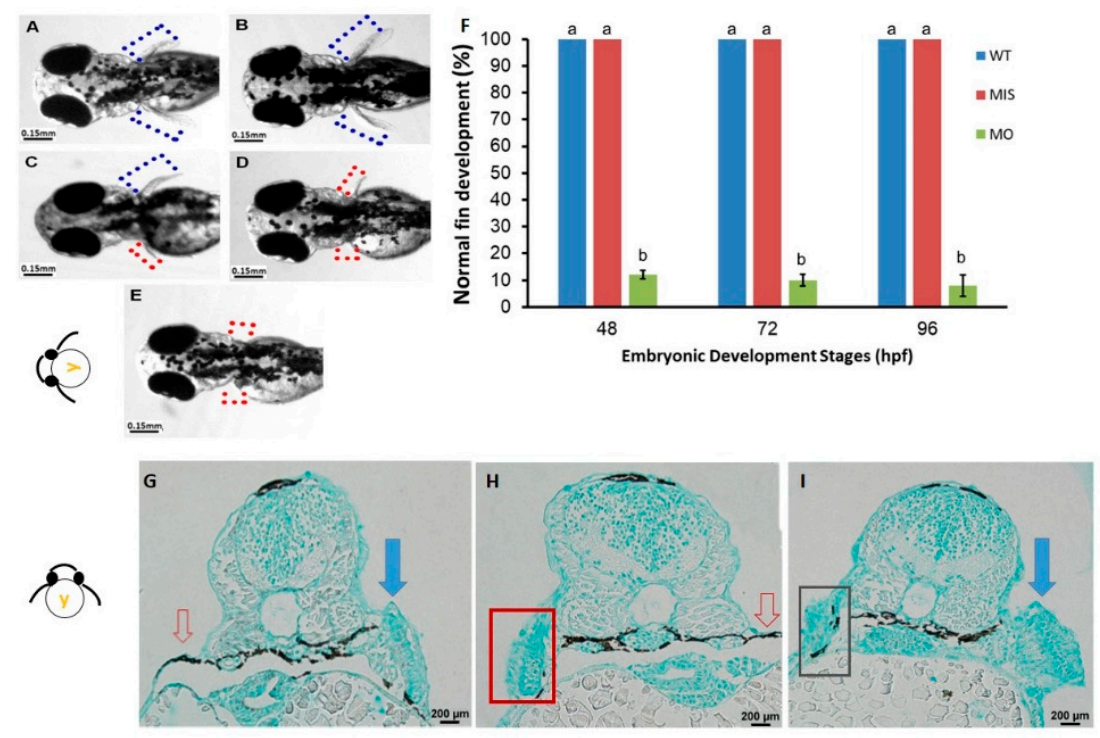

Figure 1. Phenotypes of pectoral fins in $t b x 5 a$-deficient embryos. Wild-type embryos with bilateral normal development of the pectoral fin (A,B). Unilateral hypoplasia (C), unilateral agenesis (D), and bilateral agenesis (E) of the pectoral fin in $t b x 5 a$ morphants at $96 \mathrm{hpf}$. The rate of normal fin development was significantly reduced in $t b x 5 a$-deficient embryos at 48, 72, and 96 hpf (F). a, b: A significant difference was detected by one-way ANOVA with Duncan's multiple range test. WT: Uninjected group, MIS: Control MO-injected group, MO: tbx $5 a$ morphant group. (G) Cross section of embryos with morphologic unilateral agenesis of the pectoral fin with Alcian blue staining showing well-organized glycosaminoglycans (GAGs) in the normal pectoral fin (blue arrow) with complete absence of chondrocytes and GAGs at the aplasia site (red arrow). (H) Cross section of the embryo with morphologic bilateral hypoplasia/agenesis of the pectoral fin showing the absence of chondrocytes and GAGs at the aplasia site (red arrow) with disorganized GAGs in the hypoplastic pectoral fin (red box). (I) Cross section of the embryo with morphologic unilateral hypoplasia of the pectoral fin showing disorganized GAGs in the hypoplastic pectoral fin (black box) and well-organized GAGs in the normal pectoral fin (blue arrow).

To rule out the possibility that pectoral fin anomalies were induced by microinjection or a nonspecific off-target effect of $t b x 5 a \mathrm{MO}$, we performed early microinjection of missense $t b x 5 a$ (Control MO-injected group) into WT embryos at the 1-4 cell stage as a control. All of these embryos showed normal pectoral fins without anomalies (50/50, 100\%, $n=50$ each, triplet) (Figure 1F). This result suggested that the possibility of an off-target effect of $t b x 5 a \mathrm{MO}$ inducing anomalies during fin development in this study is negligible.

\subsection{Depressed tbx5a Gene Expression}

At $48 \mathrm{hpf}, t b x 5 a$ expression was restricted to the pectoral fin buds, eyes, and heart (Figure 2A,C). At $48 \mathrm{hpf}$, high levels of $t b x 5 a$ expression were detected throughout the fin buds. Expression of $t b x 5 a$ in the distal part of the pectoral fin buds was more intense than that in the proximal region (Figure 2A,C). Moreover, $t b x 5 a$ expression in pectoral fins was significantly reduced in the $t b x 5 a$ morphant at $48 \mathrm{hpf}$ (Figure 2B,D). 


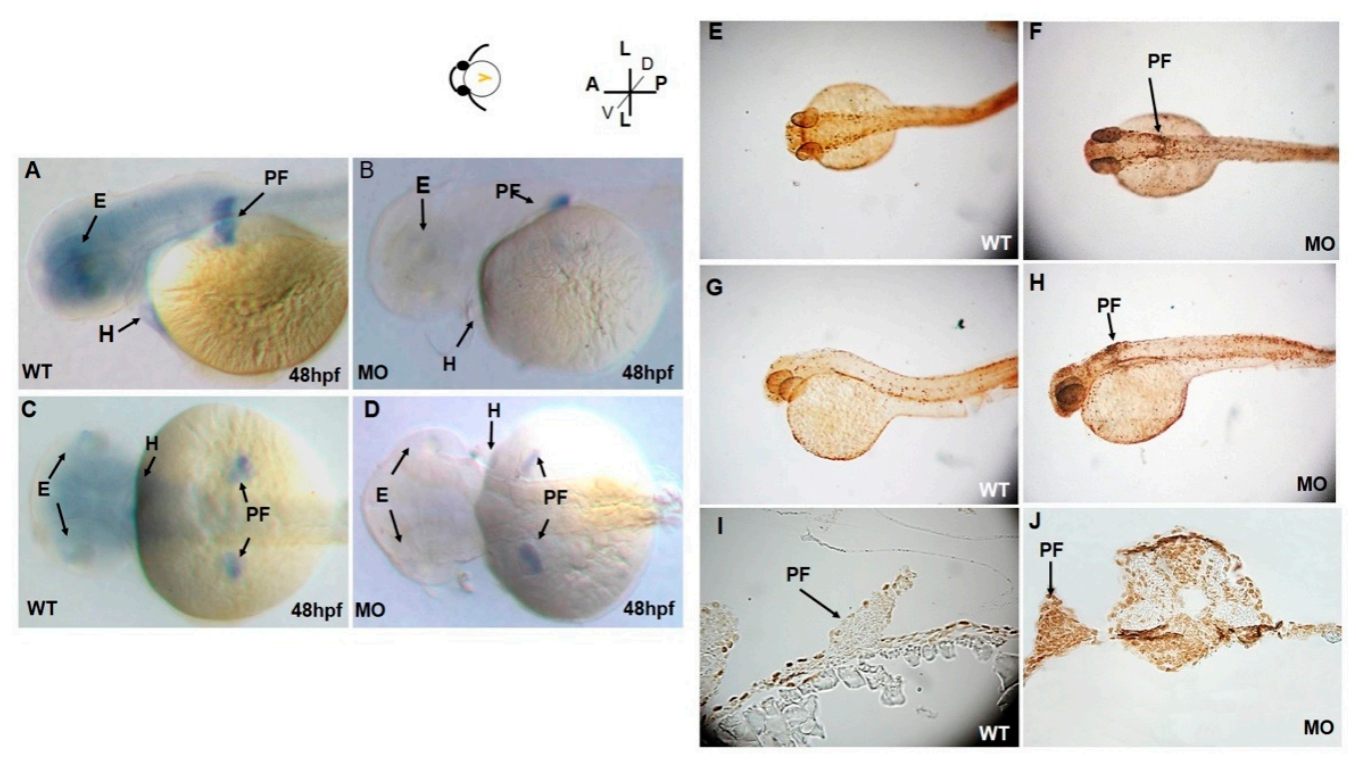

Figure 2. Lateral (A) and dorsal (C) views of $t b x 5 a$ gene expression in the eyes, heart, and bilateral pectoral fins of wild-type embryos at $48 \mathrm{hpf}$. tbx $5 a$ gene expression in the eyes, heart, and pectoral fins was severely inhibited at $48 \mathrm{hpf}$ in both the lateral (B) and ventral (D) views. Detection of apoptotic cells by the TUNEL assay in wild-type $(\mathbf{E}, \mathbf{G}, \mathbf{I})$ and $t b x 5 a$ knockdown $(\mathbf{F}, \mathbf{H}, \mathbf{J})$ zebrafish embryos at $37 \mathrm{hpf}$. No TUNEL-positive cells are observable in the dorsal view (E), lateral view (G), or cross sections at the pectoral fin level (I) in wild-type embryos. However, massive numbers of TUNEL-positive cells in the pectoral fin region (black arrow) in the $t b x 5 a$ morphant embryos were visible in the dorsal view $(\mathbf{F})$, lateral view $(\mathbf{H})$ and cross section at the pectoral fin level $(\mathbf{J})$. WT, Uninjected group; MO, tbx5a morphant group; PF, pectoral fin; E, eye, $\mathrm{H}$, heart.

\subsection{Microarray Screening}

We used a zebrafish chip (DNA microarray) to screen pectoral fin development-related gene transcripts. The total number of screened gene transcripts was 43,663 , and the cutoff point was a 1.5-fold change in expression. After knockdown of $t b x 5 a, 2250$ gene transcripts were upregulated at $24 \mathrm{hpf}$, 2493 were upregulated at $30 \mathrm{hpf}$, and 2601 were upregulated at $48 \mathrm{hpf}$. After knockdown of $t b x 5 a$, 1248 gene transcripts were downregulated at $24 \mathrm{hpf}, 747$ were downregulated at $30 \mathrm{hpf}$, and 1468 were downregulated at $48 \mathrm{hpf}$.

Microarrays identified 18 downregulated gene transcripts related to fin development in the tbx 5 morphant group at 24 and $30 \mathrm{hpf}$, but none of the gene transcripts related to cartilage development were upregulated in the tbx 5 morphant group. The 18 downregulated gene transcripts comprised the following: 3 fin morphogenesis-related gene transcripts-cx43 (connexin 43 , which is related to transmembrane transport activity and gap junction alpha-1 protein), bbs7 (Bardet-Biedl syndrome 7 is a protein-coding gene, related pathways are organelle biogenesis and maintenance and cargo trafficking to the periciliary membrane), and hoxc8a (homeobox C8a is a DNA-binding gene related to embryonic pectoral fin morphogenesis); 3 fin development-related gene transcripts-hhip (hedgehog interacting protein encoding gene, an important morphogen for anteroposterior patterns of limbs), axin 1 (encodes a cytoplasmic protein that contains a G-protein signaling regulation domain and a disheveled and axin domain), and $m s x b$ (the encoded protein of muscle segment homeobox B is a transcriptional repressor whose normal activity may establish a balance between the survival and apoptosis of neural crest-derived cells required for proper craniofacial morphogenesis); and 12 cartilage development-related gene transcripts-mmp14a (the protein encoded by matrix metalloproteinase 14 alpha is involved in the breakdown of ECM in embryonic development and tissue remodeling), $\sec 23 b$ (Sec23 homolog B is an essential component of coat protein complex II-coated vesicles that transport secretory proteins from the endoplasmic reticulum to the Golgi 
complex), tfap $2 a$ (transcription factor AP-2 alpha is a protein-coding gene, and defects in this gene are a cause of branchio-oculo-facial syndrome), slc35b2 (solute carrier family 35; member B2 is a protein-coding gene, and the related pathways are metabolism and glycosaminoglycan metabolism), $d l x 5 a$ (distal-less homeobox gene $5 \mathrm{a}$ is related to embryonic viscerocranium morphogenesis and split-hand/foot malformation 1 with sensorineural hearing loss), dlx1a (distal-less homeobox gene $1 \mathrm{a}$ is related to the pharyngeal arch cartilage that forms the largest skeletal element of the ventral branchial arches), $\operatorname{tfap} 2 b$ (transcription factor AP-2 beta is related to the stimulation of cell proliferation and suppression of the terminal differentiation of specific cell types during embryonic development), fmr1 (fragile $\mathrm{X}$ mental retardation 1 is related to multifunctional polyribosome-associated RNA-binding protein, which plays a central role in neuronal development and synaptic plasticity), run $x 3$ (runt-related transcription factor 3 is a protein-coding gene and related to cleidocranial dysplasia), cdh2 (cadherin 2, a neuronal gene encoding a classical cadherin and a member of the cadherin superfamily, plays a role in the formation of cartilage and bone), lect1 (leukocyte cell-derived chemotaxin 1 is expressed in the avascular zone of prehypertrophic cartilage, and its expression decreases during chondrocyte hypertrophy and vascular invasion), acvr2a (activin receptor IIa encodes a receptor that mediates the functions of activins, which are members of the transforming growth factor-beta (TGF-beta) superfamily involved in diverse biological processes), and mmp14b (matrix metalloproteinase 14 (membrane-inserted) beta MMP14 (matrix metalloproteinase 14), an essential protein-coding gene for pericellular collagenolysis and the modeling of skeletal and extraskeletal connective tissues during development) (Table 1). 
Table 1. Cartilage and fin development-related gene transcripts downregulated 1.5x in tbx5 knockdown embryos in different embryonic developmental stages.

\begin{tabular}{|c|c|c|c|c|c|}
\hline Genbank Accession & Gene Symbol & Gene Name & Biological Process & Log2 Expression Ratio & Stages \\
\hline NM_131038 & $c x 43$ & connexin 43 & fin development & -1.98 & 24 \\
\hline NM_001080012 & hhip & hedgehog interacting protein & fin development & -1.63 & 24 \\
\hline NM_001077145 & bbs7 & Bardet-Biedl syndrome 7 & fin development & -2.02 & 24 \\
\hline NM_194416 & $m m p 14 a$ & matrix metalloproteinase 14 (membrane-inserted) alpha & cartilage development & -1.67 & 24 \\
\hline NM_199777 & $\sec 23 b$ & Sec23 homolog B (S. cerevisiae) & cartilage development & -1.88 & 24 \\
\hline NM_176859 & tfap $2 a$ & transcription factor AP-2 alpha & cartilage development & -2.12 & 24 \\
\hline NM_131503 & $\operatorname{axin} 1$ & axin 1 & fin development & -1.71 & 30 \\
\hline NM_131260 & $m s x b$ & muscle segment homeobox B & fin development & -1.61 & 30 \\
\hline NM_001005771 & hoxc8a & homeo box C8a & fin development & -1.93 & 30 \\
\hline NM_205635 & slc35b2 & solute carrier family 35 , member B2 & cartilage development & -1.89 & 30 \\
\hline NM_131306 & $d l x 5 a$ & distal-less homeobox gene $5 a$ & cartilage development & -1.52 & 30 \\
\hline NM_131305 & $d l x 1 a$ & distal-less homeobox gene 1a & cartilage development & -1.84 & 30 \\
\hline NM_001024665 & tfap $2 b$ & transcription factor AP-2 beta & cartilage development & -1.77 & 30 \\
\hline NM_152963 & fmr1 & fragile $\mathrm{X}$ mental retardation 1 & cartilage development & -1.53 & 30 \\
\hline BC163560 & runx3 & runt-related transcription factor 3 & cartilage development & -1.65 & 30 \\
\hline NM_131081 & $c d h 2$ & cadherin 2 , neuronal & cartilage condensation & -1.94 & 30 \\
\hline NM_001126448 & lect1 & leukocyte cell derived chemotaxin 1 & cartilage development & -1.75 & 30 \\
\hline NM_001110278 & acvr $2 a$ & activin receptor IIa & cartilage development & -1.99 & 30 \\
\hline NM 194414 & $m m p 14 b$ & matrix metalloproteinase 14 (membrane-inserted) beta & cartilage development & -1.87 & 30 \\
\hline
\end{tabular}




\subsection{Chondrocytes and Glycosaminoglycans in Pectoral Fins}

HE and Alcian blue staining were used in histological sections of pectoral fins to reveal chondrocytes (Figure 3) and glycosaminoglycans (GAGs) in the cartilage (Figure 4). The apical thickening, apical fold, and beginning of the central CC in the pectoral fin could be observed in WT embryos at $37 \mathrm{hpf}$ (Figure 3A). Further conversion of the CC to a tubular shape surrounded by ventral and dorsal myogenic mesenchyme was observed at $48 \mathrm{hpf}$ (Figure 3C), and the formation of an endoskeletal disc with dorsal and ventral musculature was observed at $72 \mathrm{hpf}$ (Figure 3E). The chondrocyte number was reduced (Figure 3B), and CC was severely depressed in the $t b x 5 a$ morphant group at 37 hpf (Figure 3D). The formation of tubular-shaped cartilage tissue was barely visible at $48 \mathrm{hpf}$ (Figure 3F).

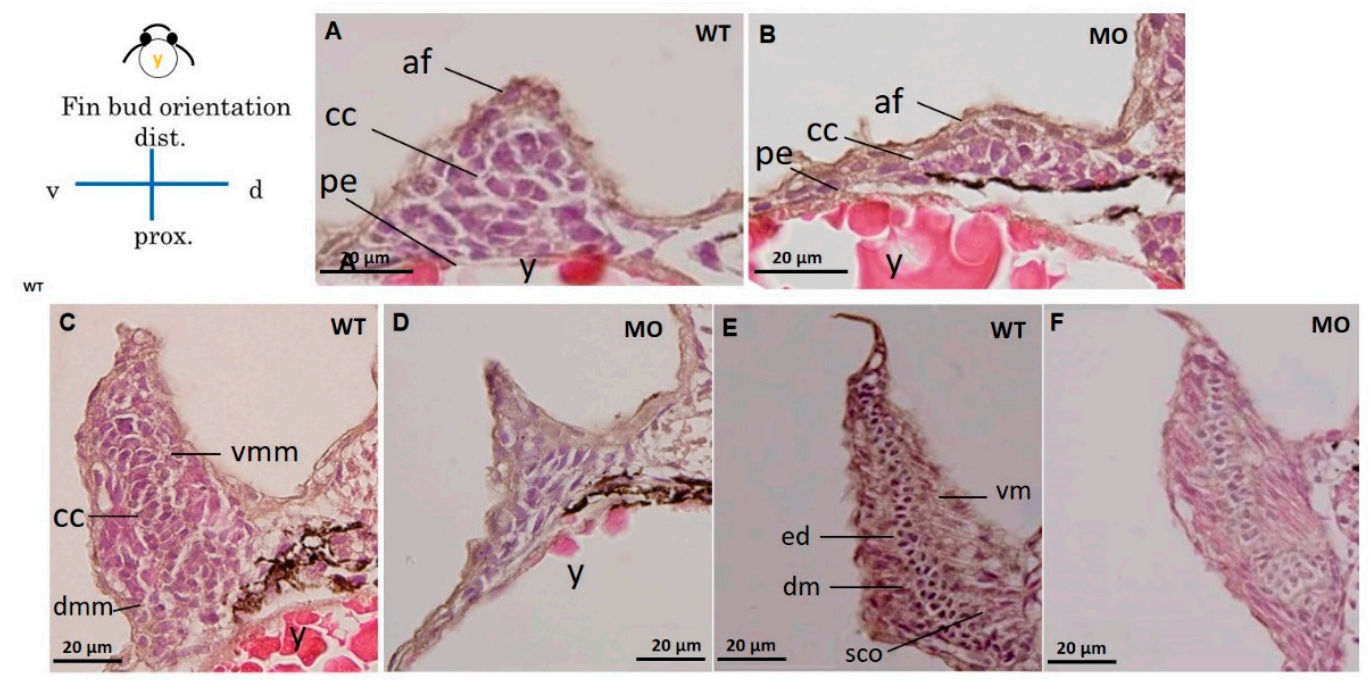

Figure 3. Cross section of a pectoral fin stained with HE. Wild-type embryos with well-developed myocytes and organized chondrocytes in pectoral fins at 37 (A), 48 (C), and $72 \mathrm{hpf}(\mathbf{E})$. The hypoplastic pectoral fin of the $t b x 5 a$ morphant showed reduced myocytes and disorganized chondrocytes at 37 (B), 48 (D) and $72 \mathrm{hpf}$ (F). (af, apical fold; cc, chondrogenic condensation; dm, dorsal musculature; dmm, dorsal myogenic mesenchyme; ed. endoskeleton disc; vm, ventral musculature; vmm, ventral myogenic mesenchyme; pe, peritoneal epithelium; y, yolk, WT, Uninjected group, MO, tbx5 morphant group).

Compared with the Uninjected group (Figure 4A,C,E), the tbx5 morphant group showed a reduction in GAGs in the cartilage (Figure 4B,D,F). High levels of GAGs were evenly distributed throughout the whole pectoral fin in WT embryos in the early stage of CC at $37 \mathrm{hpf}$ (Figure 4A). By contrast, GAGs were sparse in the tbx5 morphant group (Figure 4B). Synchronized with the late phase of CC at $48 \mathrm{hpf}$, abundant GAGs were located in the central region of pectoral fins in the Uninjected group (Figure 4C). By contrast, GAGs were formed only loosely in the tbx5 morphant group at 48 hpf (Figure 4D). At the stage of endoskeleton formation, high levels of GAGs were concentrated centrally in the fin in the Uninjected group at $72 \mathrm{hpf}$ (Figure $4 \mathrm{E}$ ). The stunted pectoral fins in the $t b x 5 a$ morphant group appeared substantially thickened with variable shortening of the proximal-distal length (Figure $4 \mathrm{~F}$ ). The endoskeletal disc in stunted pectoral fins showed multiple layers of disorganized chondrocytes with hypoplastic muscular development (Figures $3 \mathrm{~F}$ and $4 \mathrm{~F}$ ). The embryo with morphological bilateral hypoplasia/agenesis of the pectoral fin showed an absence of chondrocytes and GAGs at the aplasia site, disorganized GAGs in the hypoplastic pectoral fin (Figure 1G,H), and well-organized GAGs in the normal pectoral fin (Figure 1H,I). 


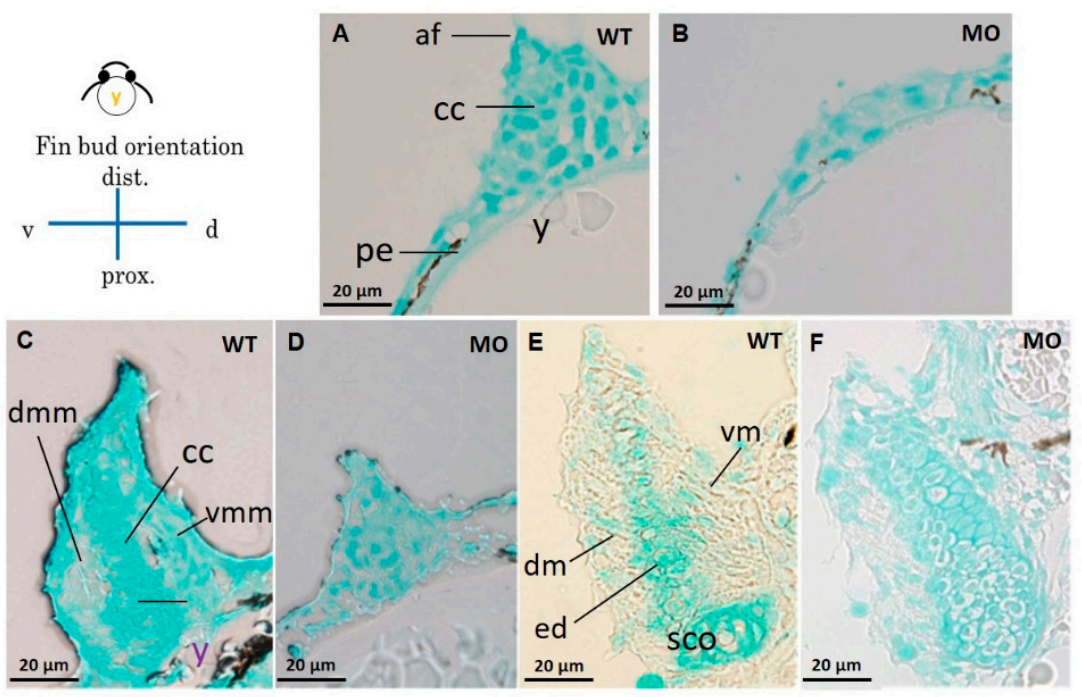

Figure 4. Cross section of pectoral fins stained with Alcian blue. Wild-type embryos showed well-developed apical folds and organized chondrocytes at 37 (A), 48 (C), and $72 \mathrm{hpf}(\mathbf{E})$. The $t b x 5 a$ morphant showed severe pectoral fin hypoplasia with loss of the apical fold, reduced chondrocytes, unorganized chondrogenic condensation and endoskeletal disc at 37 (B), 48 (D), and $72 \mathrm{hpf}$ (F). (af, apical fold; cc, chondrogenic condensation; dm, dorsal musculature; dmm, dorsal myogenic mesenchyme; ed. endoskeleton disc; vm, ventral musculature; vmm, ventral myogenic mesenchyme; pe, peritoneal epithelium; y, yolk).

\subsection{Reduced cdh2 Gene Expression}

After microinjection of $19.4 \mathrm{ng} / 2.3 \mathrm{~nL}$ tbx $5 a \mathrm{MO}$ into zebrafish embryos at the 1-4 cell stage, embryos were collected at 26, 30,37, and $48 \mathrm{hpf}(n=50$, triplet). Compared with the Uninjected group, the $t b x 5 a$ morphant group showed a significant reduction in $c d h 2$ expression at 26, 30, 37, and $48 \mathrm{hpf}$ as determined by RT-qPCR (Figure 5G).
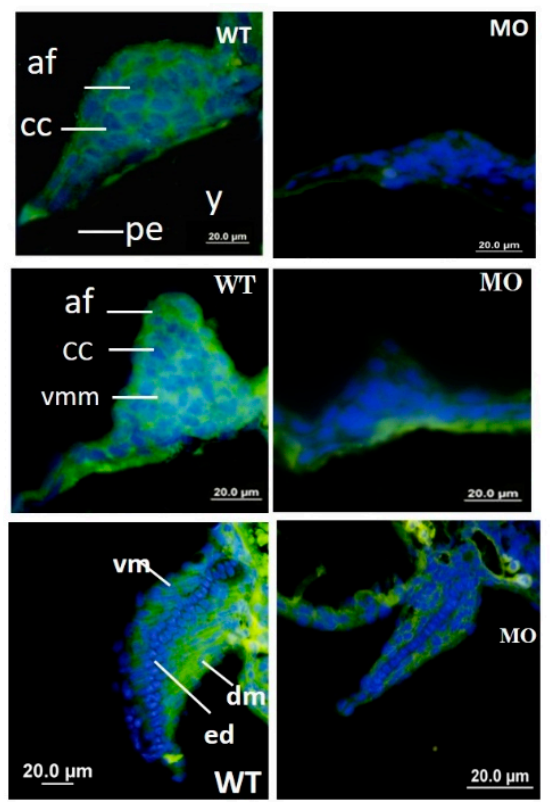
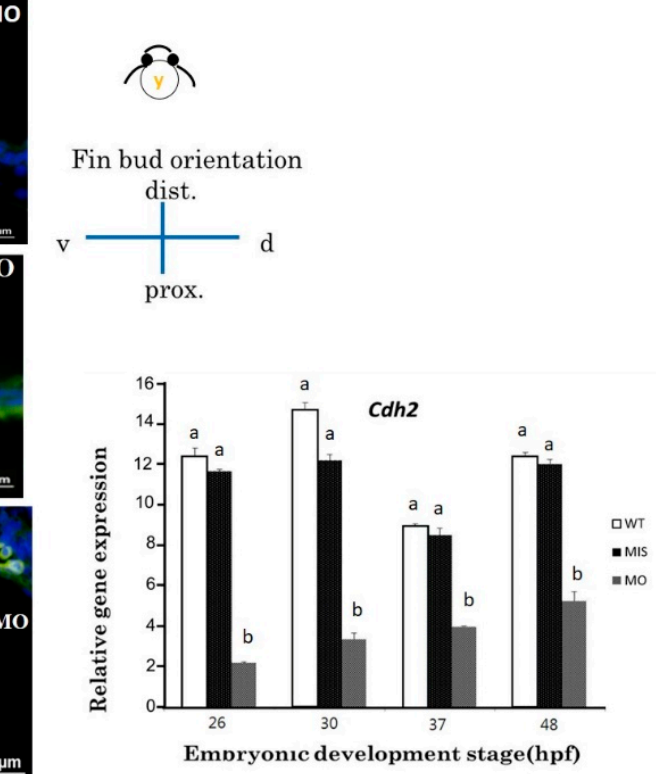

Figure 5. Cross sections of pectoral fins subjected to immunofluorescence staining for CDH2. Pectoral fins of wild-type embryos showed abundant N-cadherin surrounding chondrocytes at 37 (A) and $48 \mathrm{hpf}(\mathrm{C})$. N-cadherin is observable in the dorsal and ventral muscular region surrounding 
the endoskeletal disc at $72 \mathrm{hpf}(\mathrm{E})$. The $t b x 5 a$ morphant with a stubby pectoral fin showed a lack of $\mathrm{N}$-cadherin at $37 \mathrm{hpf}(\mathbf{B}), 48 \mathrm{hpf}(\mathbf{D})$ and $72 \mathrm{hpf}(\mathbf{F})$. cdh2 expression was significantly inhibited in the tbx5 morphant group at 26,30,37, and $48 \mathrm{hpf}(\mathrm{G})$ ( $n=50$ embryos, triplet). (cl, cleithrum; cc, chondrogenic condensation; dm, dorsal musculature; ed, endoskeleton disc; sco, scapulocoracoid; vm, ventral musculature; blue, nuclear; green, $\mathrm{CDH} 2$ protein, WT: Uninjected group, MIS: Control MO-injected group, MO: tbx5 morphant group, a, b: A significant difference was detected by one-way ANOVA with Duncan's multiple range test.

\subsection{Reduction in N-Cadherin Protein Expression in Pectoral Fins}

We used immunostaining to assess the localization of the N-cadherin protein (CDH2) in thin sections of pectoral fins. The formation of $\mathrm{N}$-cadherin at $37 \mathrm{hpf}$ was severely affected in the $t b \times 5 a$ morphant group compared with that in the control group, resulting in a severely hypoplastic pectoral fin (Figure 5). At the CC stage, the level of $\mathrm{N}$-cadherin in the Uninjected group extended homogenously throughout the pectoral fin tissue at $37 \mathrm{hpf}$ (Figure 5A), but only sparse $\mathrm{N}$-cadherin was found in the $t b x 5 a$ morphant group (Figure $5 \mathrm{~B}$ ). The levels of $\mathrm{N}$-cadherin synchronized with the development of the dorsal and ventral myogenic mesenchymal mesoderm in the Uninjected group and extended homogenously throughout the pectoral fin tissue at $48 \mathrm{hpf}$ (Figure 5C). In the tbx5a morphant group, the formation of $\mathrm{N}$-cadherin was depressed and restricted in only the basement part of the pectoral fin at $48 \mathrm{hpf}$ (Figure 5D). At the endoskeleton formation stage at $72 \mathrm{hpf}, \mathrm{N}$-cadherin extended homogenously from the dorsal muscular structure to the trunk in the Uninjected group (Figure 5E). Both the ventral and dorsal muscular structures surrounding the endoskeleton myogenic mesenchyme exhibited high levels of N-cadherin in the Uninjected group (Figure 5F). The centrally located cartilage lacked $\mathrm{N}$-cadherin and was well-formed at $72 \mathrm{hpf}$ in the Uninjected group. Nevertheless, the perichondrial cells surrounding the forming cartilage in the Uninjected group exhibited high levels of $\mathrm{N}$-cadherin (Figure 5E). The weak expression of N-cadherin could be detected in the stunted fins in the $t b x 5 a$ morphant group at $72 \mathrm{hpf}$ (Figure 5F).

\subsection{Reduction in ECM Protein and Cx4 Expression}

HA-binding protein and fibronectin in the pectoral fin were examined by immunostaining at $48 \mathrm{hpf}$. HA-binding protein (Figure 6A) and fibronectin (Figure 6C) were strongly expressed throughout the pectoral fin in the Uninjected group, but their expression levels were significantly decreased in the $t b x 5 a$ morphant group (Figure $6 \mathrm{~B}, \mathrm{D}$ ). Using real-time RT-PCR, we confirmed that $C \times 43$ was significantly downregulated in the $t b x 5 a$ morphant group throughout different stages of pectoral fin formation (Figure 6E).
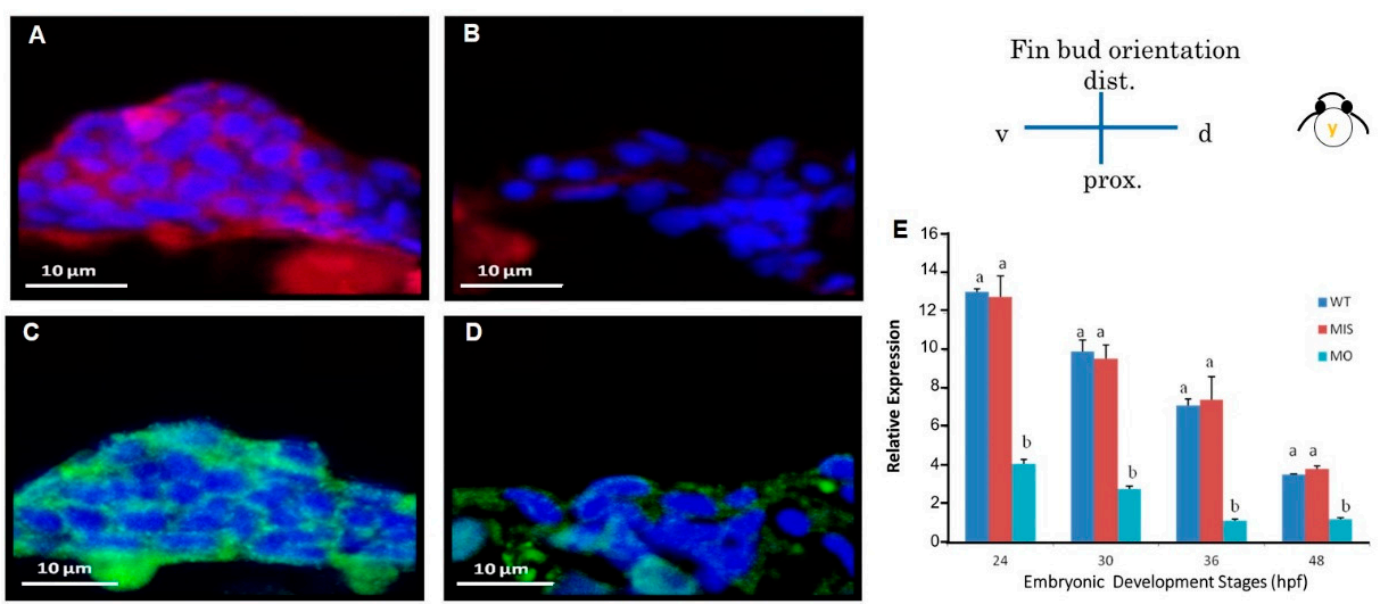

Figure 6. The immunochemical characterization of fibronectin and HA-binding protein in fin tissue at 48 hpf. Compared with wild-type embryos (A,C), tbx5a MO embryos showed decreased expression 
of fibronectin and HA-binding protein at $48 \mathrm{hpf}(\mathbf{B}, \mathbf{D})$. C $x 43$ expression in the $t b x 5 a$ morphant group was significantly inhibited at 24, 30,36, and $48 \mathrm{hpf}(n=50$, triplet) (E). a, b: A significant difference was detected by One-way ANOVA with Duncan's multiple range test; red in (A,B), fibronectin; green in (C,D), HA-binding protein; blue: nuclear, WT: Uninjected group, MIS: Control MO-injected group, MO: tbx 5 a morphant group.

\subsection{Increase in Apoptosis-Related Proteins in Pectoral Fins}

The terminal deoxynucleotidyl transferase dUTP nick end labeling (TUNEL) assay demonstrated only a few apoptotic cells in the Uninjected group at $37 \mathrm{hpf}$ (Figure 2E,G,I). However, a marked increase in apoptotic cells was observed in the head, heart, fin, and spine in the tbx5a morphant group (Figure 2F,H,J).

Two apoptosis-related proteins (B cell lymphoma 2 (BCL2) and BCL2-associated death promoter (BAD)) were examined by immunostaining at $30 \mathrm{hpf}$. Our results showed a remarkable increase in BCL2 and BAD protein expression in the tbx $5 a$ morphant group (Figure $7 \mathrm{~B}, \mathrm{D}$ ) compared with that in the Uninjected group (Figure 7A,C). Using real-time RT-PCR, we confirmed that BAD (Figure 7E) and BCL2 (Figure 7F) were significantly upregulated in the $t b x 5 a$ morphant group throughout the different stages of pectoral fin formation.
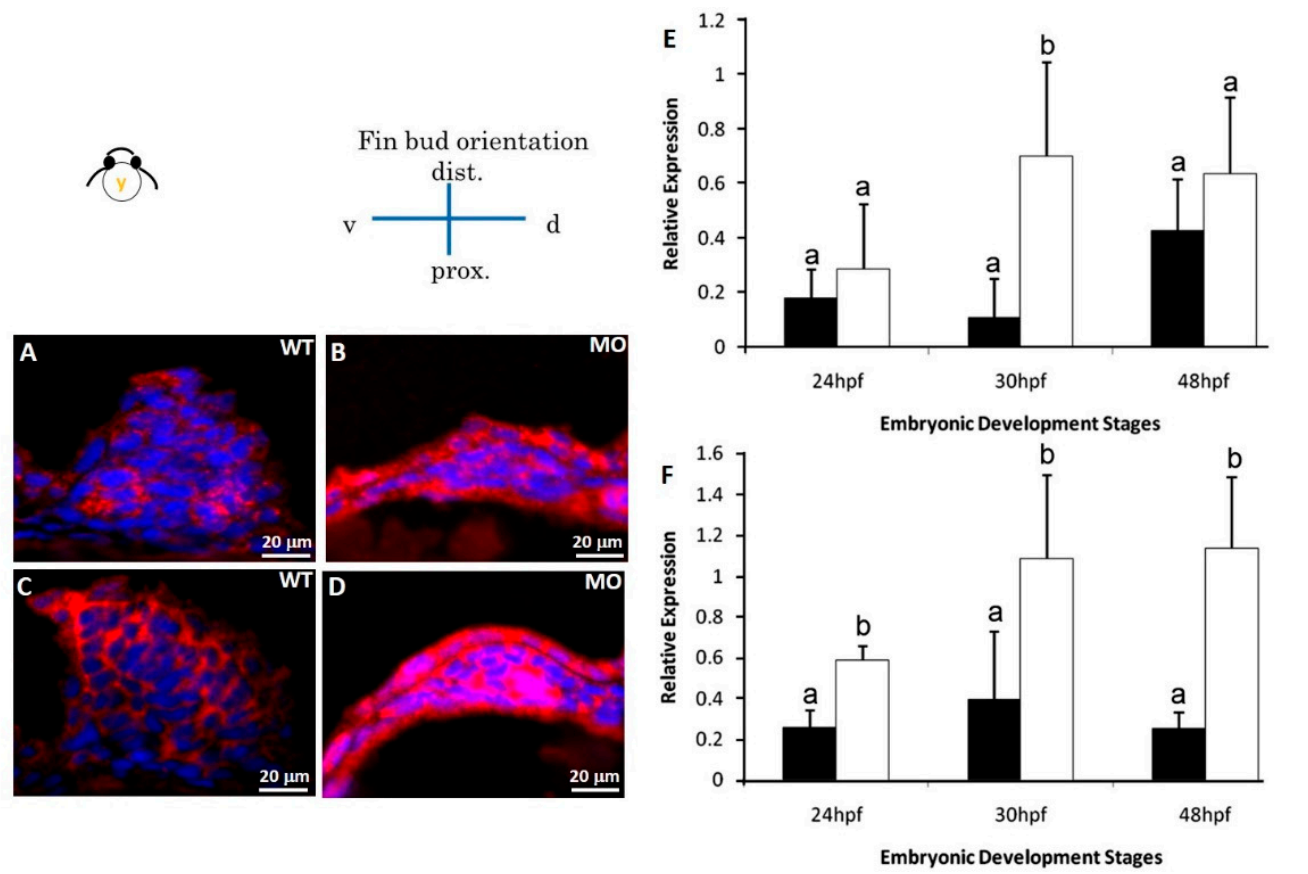

-WT

$\square \mathrm{MO}$

Figure 7. Immunohistochemical staining of apoptosis-related proteins. Pectoral fins were stained with apoptosis-related (BAD and BCL2) antibodies (red) and counterstained with DAPI (blue) to observe the nucleus. BAD (B) and BCL2 (D) proteins were significantly uninhibited in the tbx5a morphant group compared with those in the Uninjected group $(\mathbf{A}, \mathbf{C})$. The embryo anterior is shown on the left. The Bad (E) and Bcl2 (F) expression in the tbx5a morphant group was significantly uninhibited at 24, 30,36 , and $48 \mathrm{hpf}(n=50$, triplet) (E). hpf, hours postfertilization; WT, Uninjected group; MO, tbx $5 a$ morphant group. a, b: A significant difference was detected by a, b: A significant difference was detected by One-way ANOVA with Duncan's multiple range test.

\section{Discussion}

Cartilage formation in developing vertebrate embryonic limbs consists of a series of events involving mesenchymal cell recruitment, migration, proliferation, and condensation [24]. CC is the critical stage in the development of cartilage and other mesenchymal tissues [1]. 
Genetic mutations, such as brachypod and phocomelia in mice and talpid in chickens, cause abnormal chondrogenesis during the CC stage and skeletal defects $[25,26]$. In this study, $t b x 5 a$ knockdown resulted in early-onset alterations in chondrogenesis from mesenchymal cell migration onward. One of the earliest events in chondrogenesis in the pre-CC stage is the aggregation of chondroprogenitor mesenchymal cells. The microarray screening results in this report provided strong evidence that several genes involved in chondrogenesis were downregulated in the $t b x 5 a$ morphant group at 24 and $30 \mathrm{hpf}$. A high cell density of chondroprogenitor mesenchymal cells is required for the occurrence of chondrogenesis, and the extent of cellular condensation correlates with the level of chondrogenesis [27]. In our study, the mesenchymal cell density of pectoral fins in larvae at the CC stage was high in the Uninjected group and severely reduced in the $t b x 5 a$ morphant group. As demonstrated by our previous study, tbx5a deficiency provokes the expression of apoptosis-related genes distributed across multiple organs, reducing the promising maturation of tbx5a morphant embryos [28]. Using TUNEL and immunostaining assays, increases in apoptosis (BAD and BCL2)-related proteins were observed in pectoral fins in the $t b x 5 a$ morphant group at the CC stage ( 30 and $37 \mathrm{hpf}$ ). Our findings suggested that mesenchymal cell reduction in the tbx5a morphant was related to cell apoptosis, which subsequently interfered with the morphogenesis of the pectoral fin and contributed to its hypoplasia or even agenesis in the $t b x 5 a$ morphant group.

The cartilage ECM of zebrafish larvae is a polymorphic structure consisting of collagen I, collagen II, and large networks of PGs that contain GAGs, HA, fiber, and other molecular components, including fibronectin and laminin [29,30].

GAGs play an indispensable role during chondrogenesis to create highly organized elements in pectoral fin formation. GAGs are important protein carrier molecules in mesenchymal cell condensation, where they are involved in a wide range of signaling processes during pectoral fin development [31]. We used Alcian blue to stain GAGs in cartilage in transverse sections of pectoral fins and observed high expression levels in all stages in the Uninjected group. However, the formation of GAGs in the $t b x 5 a$ morphant group was severely reduced at the $\mathrm{CC}$ and endoskeleton formation stages. The endoskeletal disc was well organized in the WT group at $72 \mathrm{hpf}$, but it was hypoplastic and disorganized with residual GAGs in the $t b x 5 a$ morphant group.

We identified two GAG-related genes ( $s l c 35 B 2$ and $c d h 2)$ that were downregulated in the $t b x 5 a$ morphant group via microarray analysis. We report, here, for the first time that $t b x 5$ gene expression affects $c d h 2$ gene expression and $\mathrm{N}$-cadherin formation. In zebrafish, $\mathrm{N}$-cadherin, encoded by the gene $c d h 2$, is expressed extensively from late blastula stages onward [13]. $c d h 2$ function is crucial for the normal chondrogenesis of zebrafish pectoral limb buds by mediating functional mesenchymal cell condensation [32]. CC in the endoskeleton in $c$ dh 2 knockdown embryos is missing or barely recognizable in the zebrafish fin bud [13]. These changes in the zebrafish limb bud in $c d h 2$ knockdown embryos are very similar to those in the tbx5a morphant group.

$\mathrm{N}$-cadherin is a key cell adhesion molecule for chondroprogenitor mesenchymal cell-cell adhesion at the CC stage [33-35]. A lack of $\mathrm{N}$-cadherin formation results in the failure of mesenchymal cell condensation, thus inhibiting subsequent chondrogenesis [36-39]. In this report, N-cadherin was normally present throughout the morphogenesis of the pectoral fin in the mesoderm-derived part of the paired larvae pectoral fins. High levels of N-cadherin were observed mainly at the basement of the pectoral fin bud and central trunk in the Uninjected group at the early stage of CC ( $37 \mathrm{hpf})$. These high levels of $\mathrm{N}$-cadherin extended throughout the entire fin in the late CC stage ( $48 \mathrm{hpf}$ ) and were diminished at the endoskeleton formation stage $(72 \mathrm{hpf})$. Nevertheless, the formation of $\mathrm{N}$-cadherin in the zebrafish endoskeleton in the $t b x 5 a$ morphant group was severely reduced throughout the entire CC stage. N-cadherin was completely absent in cartilage tissue at $72 \mathrm{hpf}$ in the Uninjected group, whereas the perichondria cells surrounding the formed cartilage still exhibited high $\mathrm{N}$-cadherin levels. The formation of $\mathrm{N}$-cadherin surrounding the formed cartilage in the $t b x 5 a$ morphant group was severely reduced at $72 \mathrm{hpf}$. 
The microarray screening results provided strong evidence that multiple genes related to ECM formation were downregulated in the tbx $5 a$ morphant group at the pre-CC ( $24 \mathrm{hpf}$ ) and early CC stages (30 hpf). Fibronectin is a large ECM glycoprotein that affects N-cadherin cell-cell and cell-matrix adhesions [40]. The pectoral fin disk cartilage of zebrafish is notable for its fibronectin-rich ECM [41]. We examined fibronectin in the pectoral fin at $48 \mathrm{hpf}$ and found abundant fibronectin in the Uninjected group, whereas fibronectin was significantly reduced in the $t b x 5 a$ morphant group. Our results suggested that fibronectin was strongly upregulated at the condensation and differentiation stages. Depletion of TBX5 leads to abnormal fibronectin and fibrillin formation in xenopus embryos due to alterations in cardiac cell cycle progression [42]. We previously observed a similar relationship between abnormal fibronectin and cardiac cell cycle disruption at the stage of myocardium formation in $t b x 5 a$ knockdown zebrafish [22,28].

$C \times 43$ regulates cell proliferation, fin size, and fin shape during the process of chondrogenesis in zebrafish embryos [43,44]. HA serves as a central organizing ECM molecule for binding to PGs and is molecularly and functionally downstream of $C \times 43$ during the process of cartilage morphogenesis [43]. Using DNA microarray screening and qPCR quantification, we report for the first time that $C x 43$ expression was significantly inhibited in the $t b x 5 a$ morphant group at $24,30,36$, and $48 \mathrm{hpf}$. We examined $\mathrm{HA}$ in the pectoral fin at $48 \mathrm{hpf}$ and found abundant HA in the Uninjected group, whereas HA was significantly reduced in the tbx5a morphant group.

Our findings support the conclusion that $t b x 5 a$ deficiency causes both cellular and ECM disturbances during cartilage formation in the developing zebrafish pectoral fins. $t b x 5 a$ deficiency induced the downregulation of several fin development-related genes. $t b x 5 a$ knockdown likely results in reduced expression of FGF8, FGF10, and WNT signaling $[15,17]$ which, in turn, results in reduced proliferation and migration of the mesoderm. The loss of $t b x 5 a$ reduces the mesodermal component of the fin buds, AER formation and signaling, increased apoptosis, and/or decreased immigration of mesenchymal cells from the lateral plate. The disruption of ECM formation, including fibronectin, HA, GAGs, and N-cadherin, was identified in the pre-CC stage onward.

In conclusion, the phenotypic anomalies of the pectoral fin in the tbx5a morphant group included a disruption of mesodermal cell migration and CC and interference with subsequent endoskeleton formation, which was related to the $t b x 5 a$ downregulation of a complex network of genes related to cell-cell communication and cell-matrix interactions. Thus, our results provide a context to understand the molecular and cellular processes of upper limb defects in Holt-Oram syndrome.

Supplementary Materials: The following are available online at http://www.mdpi.com/2221-3759/7/3/15/s1. The off-target effect of microinjected $t b x 5 a \mathrm{MO}$ was evaluated by longitudinal survival rate, heart rate follow-up, heart defect rate and rate of trunk deformity.

Author Contributions: J.-K.L., Conceptualization, Supervision, Methodology, Writing-Original draft preparation; T.-C.T., Methodology; H.L. Conceptualization and Supervision; K.H., Reviewing and Editing; C.-H.L., Reviewing and Editing; J.-H.L., Supervision, Data curation, Writing-Original draft preparation, Reviewing and Editing.

Funding: This work was supported by the I-Mei Co. \& VGHTPE cooperative research fund (R16001 and R16002).

Conflicts of Interest: The authors have no conflicts of interest to declare regarding the publication of this paper.

\section{References}

1. Hall, B.K.; Miyake, T. All for one and one for all: Condensations and the initiation of skeletal development. Bioessays 2000, 22, 138-147. [CrossRef]

2. Mercader, N. Early steps of paired fin development in zebrafish compared with tetrapod limb development. Dev. Growth Differ. 2007, 49, 421-437. [CrossRef] [PubMed]

3. Hasson, P.; Del Buono, J.; Logan, M.P. Tbx5 is dispensable for forelimb outgrowth. Development 2007, 134, 85-92. [CrossRef] [PubMed]

4. Hasson, P.; DeLaurier, A.; Bennett, M.; Grigorieva, E.; Naiche, L.A.; Papaioannou, V.E.; Mohun, T.J.; Logan, M.P. Tbx4 and tbx5 acting in connective tissue are required for limb muscle and tendon patterning. Dev. Cell 2010, 18, 148-156. [CrossRef] [PubMed] 
5. Basson, C.T.; Bachinsky, D.R.; Lin, R.C.; Levi, T.; Elkins, J.A.; Soults, J.; Grayzel, D.; Kroumpouzou, E.; Traill, T.A.; Leblanc-Straceski, J.; et al. Mutations in human TBX5 [corrected] cause limb and cardiac malformation in Holt-Oram syndrome. Nat. Genet. 1997, 15, 30-35. [CrossRef]

6. Gibson-Brown, J.J.; Agulnik, S.I.; Chapman, D.L.; Alexiou, M.; Garvey, N.; Silver, L.M.; Papaioannou, V.E. Evidence of a role for T-box genes in the evolution of limb morphogenesis and the specification of forelimb/hindlimb identity. Mech. Dev. 1996, 56, 93-101. [CrossRef]

7. Yano, T.; Abe, G.; Yokoyama, H.; Kawakami, K.; Tamura, K. Mechanism of pectoral fin outgrowth in zebrafish development. Development 2012, 139, 2916-2925. [CrossRef]

8. Ryan, K.; Chin, A.J. T-box genes and cardiac development. Birth Defects Res. Part C Embryo Today Rev. 2003, 69, 25-37. [CrossRef]

9. Begemann, G.; Ingham, P.W. Developmental regulation of Tbx5 in zebrafish embryogenesis. Mech. Dev. 2000, 90, 299-304. [CrossRef]

10. Lee, B.C.; Roy, S. Blimp-1 is an essential component of the genetic program controlling development of the pectoral limb bud. Dev. Biol. 2006, 300, 623-634. [CrossRef]

11. Grandel, H.; Schulte-Merker, S. The development of the paired fins in the zebrafish (Danio rerio). Mech. Dev. 1998, 79, 99-120. [CrossRef]

12. Thorsen, D.H.; Hale, M.E. Development of zebrafish (Danio rerio) pectoral fin musculature. J. Morphol. 2005, 266, 241-255. [CrossRef] [PubMed]

13. Liu, Q.; Kerstetter, A.E.; Azodi, E.; Marrs, J.A. Cadherin-1, -2, and -11 expression and cadherin-2 function in the pectoral limb bud and fin of the developing zebrafish. Dev. Dyn. Off. Publ. Am. Assoc. Anat. 2003, 228, 734-739. [CrossRef] [PubMed]

14. Garrity, D.M.; Childs, S.; Fishman, M.C. The heartstrings mutation in zebrafish causes heart/fin Tbx5 deficiency syndrome. Development 2002, 129, 4635-4645. [PubMed]

15. Ng, J.K.; Kawakami, Y.; Buscher, D.; Raya, A.; Itoh, T.; Koth, C.M.; Rodriguez Esteban, C.; Rodriguez-Leon, J.; Garrity, D.M.; Fishman, M.C.; et al. The limb identity gene Tbx5 promotes limb initiation by interacting with Wnt2b and Fgf10. Development 2002, 129, 5161-5170. [PubMed]

16. Rallis, C.; Bruneau, B.G.; Del Buono, J.; Seidman, C.E.; Seidman, J.G.; Nissim, S.; Tabin, C.J.; Logan, M.P. Tbx5 is required for forelimb bud formation and continued outgrowth. Development 2003, 130, 2741-2751. [CrossRef] [PubMed]

17. Mao, Q.; Stinnett, H.K.; Ho, R.K. Asymmetric cell convergence-driven zebrafish fin bud initiation and pre-pattern requires Tbx5a control of a mesenchymal Fgf signal. Development 2015, 142, 4329-4339. [CrossRef] [PubMed]

18. Ahn, D.G.; Kourakis, M.J.; Rohde, L.A.; Silver, L.M.; Ho, R.K. T-box gene tbx5 is essential for formation of the pectoral limb bud. Nature 2002, 417, 754-758. [CrossRef] [PubMed]

19. Agarwal, P.; Wylie, J.N.; Galceran, J.; Arkhitko, O.; Li, C.; Deng, C.; Grosschedl, R.; Bruneau, B.G. Tbx5 is essential for forelimb bud initiation following patterning of the limb field in the mouse embryo. Development 2003, 130, 623-633. [CrossRef]

20. Rodriguez-Esteban, C.; Tsukui, T.; Yonei, S.; Magallon, J.; Tamura, K.; Izpisua Belmonte, J.C. The T-box genes Tbx4 and Tbx5 regulate limb outgrowth and identity. Nature 1999, 398, 814-818. [CrossRef]

21. Tsai, T.C.; Lu, J.K.; Choo, S.L.; Yeh, S.Y.; Tang, R.B.; Lee, H.Y.; Lu, J.H. The paracrine effect of exogenous growth hormone alleviates dysmorphogenesis caused by tbx5 deficiency in zebrafish (Danio rerio) embryos. J. Biomed. Sci. 2012, 19, 63. [CrossRef]

22. Tsai, T.C.; Shih, C.C.; Chien, H.P.; Yang, A.H.; Lu, J.K.; Lu, J.H. Anti-apoptotic effects of IGF-I on mortality and dysmorphogenesis in tbx5-deficient zebrafish embryos. BMC Dev. Biol. 2018, 18, 5. [CrossRef]

23. Lu, J.H.; Lu, J.K.; Choo, S.L.; Li, Y.C.; Yeh, H.W.; Shiue, J.F.; Yeh, V.C. Cascade effect of cardiac myogenesis gene expression during cardiac looping in tbx5 knockdown zebrafish embryos. J. Biomed. Sci. 2008, 15, 779-787. [CrossRef] [PubMed]

24. Goldring, M.B.; Tsuchimochi, K.; Ijiri, K. The control of chondrogenesis. J. Cell. Biochem. 2006, 97, 33-44. [CrossRef] [PubMed]

25. Hall, B.K.; Miyake, T. The membranous skeleton: The role of cell condensations in vertebrate skeletogenesis. Anat. Embryol. 1992, 186, 107-124. [CrossRef] [PubMed] 
26. Mundlos, S.; Olsen, B.R. Heritable diseases of the skeleton. Part I: Molecular insights into skeletal development-transcription factors and signaling pathways. FASEB J. Off. Publ. Fed. Am. Soc. Exp. Biol. 1997, 11, 125-132. [CrossRef]

27. Lee, J.K.; Hu, J.C.; Yamada, S.; Athanasiou, K.A. Initiation of Chondrocyte Self-Assembly Requires an Intact Cytoskeletal Network. Tissue Eng. Part A 2016, 22, 318-325. [CrossRef]

28. Lu, J.; Tsai, T.; Choo, S.; Yeh, S.; Tang, R.; Yang, A.; Lee, H.; Lu, J. Induction of apoptosis and inhibition of cell growth by tbx5 knockdown contribute to dysmorphogenesis in Zebrafish embryos. J. Biomed. Sci. 2011, 18, 73. [CrossRef] [PubMed]

29. Jessen, J.R. Recent advances in the study of zebrafish extracellular matrix proteins. Dev. Biol. 2015, 401, 110-121. [CrossRef] [PubMed]

30. Govindan, J.; Tun, K.M.; Iovine, M.K. Cx43-Dependent Skeletal Phenotypes Are Mediated by Interactions between the Hapln1a-ECM and Sema3d during Fin Regeneration. PLoS ONE 2016, 11, e0148202. [CrossRef] [PubMed]

31. Michigami, T. Current understanding on the molecular basis of chondrogenesis. Clin. Pediatr. Endocrinol. 2014, 23, 1-8. [CrossRef] [PubMed]

32. Oberlender, S.A.; Tuan, R.S. Spatiotemporal profile of N-cadherin expression in the developing limb mesenchyme. Cell Adhes. Commun. 1994, 2, 521-537. [CrossRef] [PubMed]

33. Tuan, R.S. Biology of developmental and regenerative skeletogenesis. Clin. Orthop. Related Res. 2004, 427, S105-S117. [CrossRef] [PubMed]

34. Hulpiau, P.; van Roy, F. Molecular evolution of the cadherin superfamily. Int. J. Biochem. Cell Biol. 2009, 41, 349-369. [CrossRef] [PubMed]

35. Lele, Z.; Folchert, A.; Concha, M.; Rauch, G.J.; Geisler, R.; Rosa, F.; Wilson, S.W.; Hammerschmidt, M.; Bally-Cuif, L. parachute/n-cadherin is required for morphogenesis and maintained integrity of the zebrafish neural tube. Development 2002, 129, 3281-3294. [PubMed]

36. Provot, S.; Schipani, E. Molecular mechanisms of endochondral bone development. Biochem. Biophys. Res. Commun. 2005, 328, 658-665. [CrossRef] [PubMed]

37. Tuan, R.S. Cellular signaling in developmental chondrogenesis: N-cadherin, Wnts, and BMP-2. J. Bone Joint Surg. 2003, 85 (Suppl. 2), 137-141. [CrossRef]

38. Halbleib, J.M.; Nelson, W.J. Cadherins in development: Cell adhesion, sorting, and tissue morphogenesis. Genes Dev. 2006, 20, 3199-3214. [CrossRef]

39. Oberlender, S.A.; Tuan, R.S. Expression and functional involvement of N-cadherin in embryonic limb chondrogenesis. Development 1994, 120, 177-187.

40. Lefort, C.T.; Wojciechowski, K.; Hocking, D.C. N-cadherin cell-cell adhesion complexes are regulated by fibronectin matrix assembly. J. Biol. Chem. 2011, 286, 3149-3160. [CrossRef]

41. Dewit, J.; Witten, P.; Willems, M.; Huysseune, A. The distribution of fibronectin in developing zebrafish (Danio rerio) cartilage. J. Appl. Ichthyol. 2010, 26, 205-209. [CrossRef]

42. Goetz, S.C.; Brown, D.D.; Conlon, F.L. TBX5 is required for embryonic cardiac cell cycle progression. Development 2006, 133, 2575-2584. [CrossRef] [PubMed]

43. Govindan, J.; Iovine, M.K. Hapln1a is required for connexin43-dependent growth and patterning in the regenerating fin skeleton. PLoS ONE 2014, 9, e88574. [CrossRef] [PubMed]

44. Iovine, M.K.; Higgins, E.P.; Hindes, A.; Coblitz, B.; Johnson, S.L. Mutations in connexin43 (GJA1) perturb bone growth in zebrafish fins. Dev. Biol. 2005, 278, 208-219. [CrossRef] [PubMed]

(C) 2019 by the authors. Licensee MDPI, Basel, Switzerland. This article is an open access article distributed under the terms and conditions of the Creative Commons Attribution (CC BY) license (http://creativecommons.org/licenses/by/4.0/). 